Minireview

\title{
Synthesis of prenylquinones in chloroplasts
}

\author{
G. Schultz, J. Soll, E. Fiedler and D. Schulze-Siebert
}

Schultz, G., Soll, J., Fiedler, E. and Schulze-Siebert, D. 1985. Synthesis of prenylquinones in chloroplasts. - Physiol. Plant. 64: 123-129.

Additional key words - Phylloquinone, plastoquinone-9, Spinacia oleracea, tocopherol.

G. Schultz (reprint requests), E. Fiedler and D. Schulze-Siebert, Botanisches Inst., Tierärzlliche Hochschule Hannover, Bünteweg $17 d$, D-3000 Hannover 71 , West Germany, J. Soll, Botanisches Inst., Univ. München, Menzinger Straße 67, D-8000 München 19, West Germany.

\section{Introduction}

In recent studies evidence was obtained that chloroplasts of higher plants possess an autonomic system of isoprenoid synthesis. The prenyl moiety of the chloroplast isoprenoid compounds [i.e. carotenoids, chlorophylls, plastoquinones, tocopherols and phylloquinone (vitamin $\mathrm{K}_{1}$ )] originates from geranylgeranyl-PP. The present review deals with some aspects of the formation of the plastidic prenylquinones, i.e. $a$-tocopherol, plastoquinone- 9 and phylloquinone, in which the isoprenoid synthesis as well as the shikimate pathway of aromatic compound synthesis are involved. This is illustrated by the scheme in Fig. 1 which is based on results of experiments predominantly carried out with spinach chloroplasts (see Soll and Schultz 1980, Schultz et al. 1981a, 1982).

From early experiments in T. W. Goodwin's group on monocotyledons and dicotyledons it could be inferred that bicarbonate was incorporated into the abovementioned plastidic isoprenoid compounds, but not into cytoplasmatic compounds (e.g. sterols). This led to the suggestion that a direct carbon flow exists from the Calvin cycle to mevalonate and polyprenyl compound synthesis (Goodwin 1965). This idea has been strongly supported by the results of recent studies on purified, intact chloroplasts as described in the following sections.
Abbreviations (used in figures - AdoMet, $S$-adenosylmethionine; Car, Carotene; CoA-SH, Coenzyme A; DAHP, 3-deoxyD-arabinoheptulosonate-7-phosphate; HMG-CoA, 3-hydroxy3-methylglutaryl-CoA; IPP, isopentenyl-PP; Me-6-PhO $\left(\mathrm{H}_{2}\right)$, 2-methyl-6-phytylquinone (quinol); PEF, phosphoenolpyruvate; $\mathrm{PGA}, 3$-phospho-D-glycerate; $\mathrm{Phe}$, phenylalanine; $\mathrm{PQ}-9$, plastoquinone; $\delta \mathrm{T}, \delta$-tocopherol; $\delta \mathrm{TQ}, \delta$-tocopherolquinone; TLC, thinlayer chromatography; TPF, thiamine diphosphate.

The carbon flow from primary photosynthetic products to isoprenøid compounds, fatty acids and amino acids in spinach chloroplasts

From an increasing number of papers it appears conceivable that isolated chloroplasts are capable of forming isoprenoid compounds, fatty acids and amino acids from photosynthetically fixed carbon via 3phospho-D-glycerate $\rightarrow$ phosphenolpyruvate $\rightarrow$ pyruvate $\rightarrow$ acetyl-CoA. $\left[{ }^{14} \mathrm{C}\right]$-Bicarbonate was incorporated into $\beta$-carotene (Bickel and Schultz 1976) as well as into plastoquinone-9 and $\alpha$-tocopherol (Bickel et al. 1978). (For recent review on carotene synthesis in chloroplasts, see Spurgeon and Porter 1983; for reviews on prenylquinone synthesis, see Threlfall and Whistance 1971 and Pennock and Threlfall 1983.) The formation from bicarbonate of compounds derived from pyruvate (branched chain amino acids, fatty acids and isoprenoids) occurs at low rates [about $100 \mathrm{nmol}$ (mg chlorophyll) $)^{-1} h^{-1}$; Schulze-Siebert et al. 1984]. The concen- 


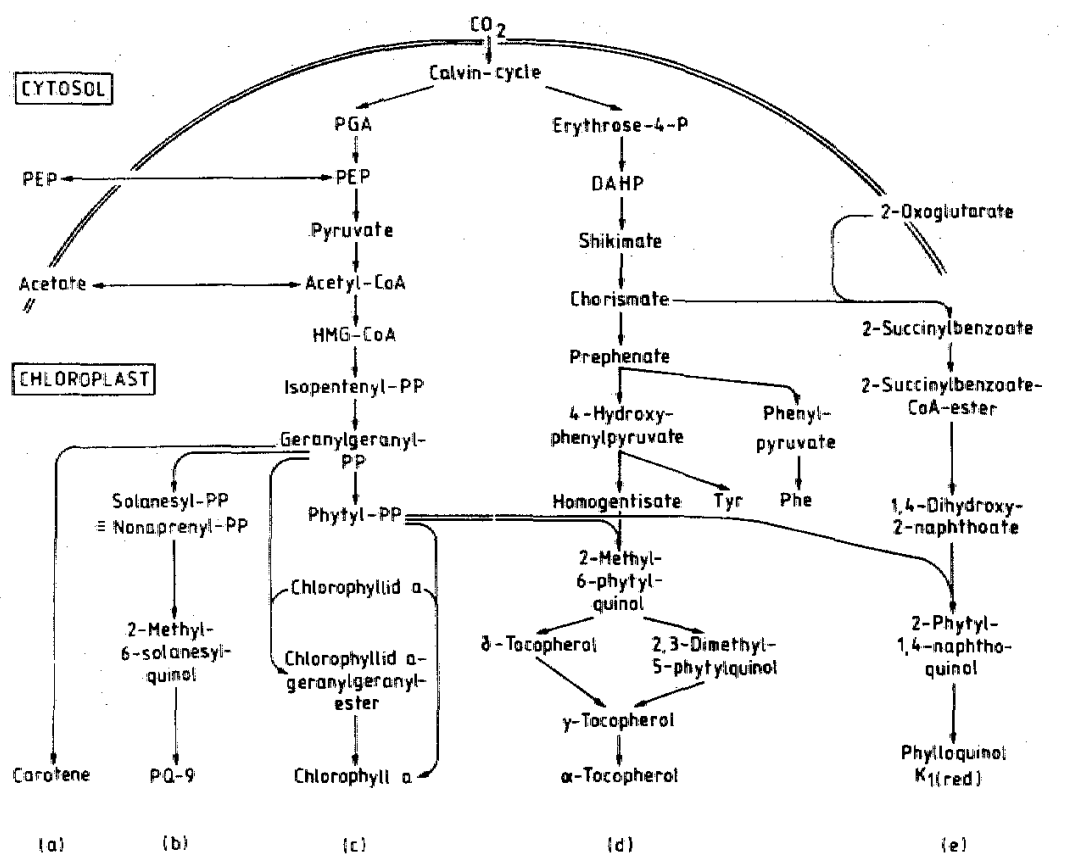

Fig. 1. Isoprenoid syntheses and accompanying reactions in chloroplasts.

a, carotenoid;

$b$, plastoguinones;

$c_{7}$ chlorophylls;

d, tocopherols;

e, phylloquinone

(vitamin $\mathrm{K}_{1}$ ).

tion of phosphoenolpyruvate and pyruvate in the osmotically impermeable space of chloroplasts (about 40 and $100 \mu \mathrm{M}$, respectively) exceeded the corresponding concentrations in the reaction medium under photosynthetic conditions about 15 times (Schulze-Siebert et al. 1984). Most of the enzymes for the formation of pyruvate from 3-phospho-D-glycerate were identified in chloroplasts (Stitt and ap Rees 1979). Pyruvate is converted by the plastidic pyruvate dehydrogenase complex (Williams and Randall 1979) into hydroxyethylthiamine diphosphate to form valine, leucine and isoleacine (for review see Miflin and Lea 1977) and into acetyl-CoA (Roughan et al. 1978) to form fatty acids (Murphy and Leech 1978, Roughan and Slack 1984) and isoprenoids... Acetoacetyl-CoA formation from acetyl-CoA has been clearly demonstrated in the stroma of purified chloroplasts (D. Schulze-Siebert, unpublished results). Pyruvate metabolism in chloroplasts occurs only at low rates of about $100 \mathrm{nmol}$ ( $\mathrm{mg}$ chlorophyll) ${ }^{-1} \mathrm{~h}^{-1}$ and is strongly regulated by cofactors and feedback of products (Schulze-Siebert et al. 1984, Homeyer et al. 1985). An additional, or under certain conditions predominant, import of phosphoenolpyruvate into chloroplasts (Buchholz and Schultz 1980, Schulze-Siebert et al: 1984) is achieved by the phosphate translocator (Heldt and Rapley 1970) of the chloroplast envelope membrane. Acetate is transferred across this membrane by diffusion and is esterified with ATP and COA-SH by the chloroplast stromal acetyl-CoA synthetase to form acetyl-CoA (Roughan et al. 1978, Stumpf et al. 1982). $\left[{ }^{14} \mathrm{C}\right]$-Bicarbonate is rapidly incorporated into aromatic amino acids (Bickel et al. 1978) at considerable rates [\$ $60 \mathrm{nmol}$ (mg chlorophyll) ${ }^{-1} \mathrm{~h}^{-1}$; Schulze-Siebert et al. 1984]. This incorporation is regulated by feedback of end-products (Bickel and Schultz 1979, Buchholz and Schultz 1980).

As is known from the studies of B. J. Miflin's group, chloroplasts are the site of the synthesis of most of the amino acids except apartate, glutamate and glycine (see Miflin and Lea 1977). Fiedler and Schultz (1984) studied the compartmentation of the shikimate pathway by fractionating protoplasts from spinach and peas into chloroplasts and extraplastidic space. The shikimate oxidoreductase-dehydroquinate bydrolyase as a bifunctional protein is solely localized in chloroplasts and is absent from the cytosol. Homogentisate is the aromatic intermediate in the formation of plastoquinone-9 and tocopherols (Whistance and Threlfall 1970; Fig. 1d and Fig. 2). It is formed from 4-hydroxyphenylpyruvate by a 4-hydroxyphenylpyruvate-dioxygenase probably associated with chloroplast envelope membranes (Fiedler et al. 1982). Thus in higher plants, the complete shikimate pathway is localized in chloroplasts. A modified pathway, probably producing pre-aromatic compounds like quinate, occurs in the cytosol (Boudet et al. 1985).

\section{On the mevalonate synthesis in chloroplasts}

Recently Arebalo and Mitchell (1984) could identify isoenzymes of HMG-CoA reductase and mevalonate ki- 
Fig. 2: Biosynthesis of tocopherols and plastoquinone-9 in chloroplasts. All reactions occur at the inner envelope membrane of chloroplasts (Soll et al. 1980a,b, 1984).

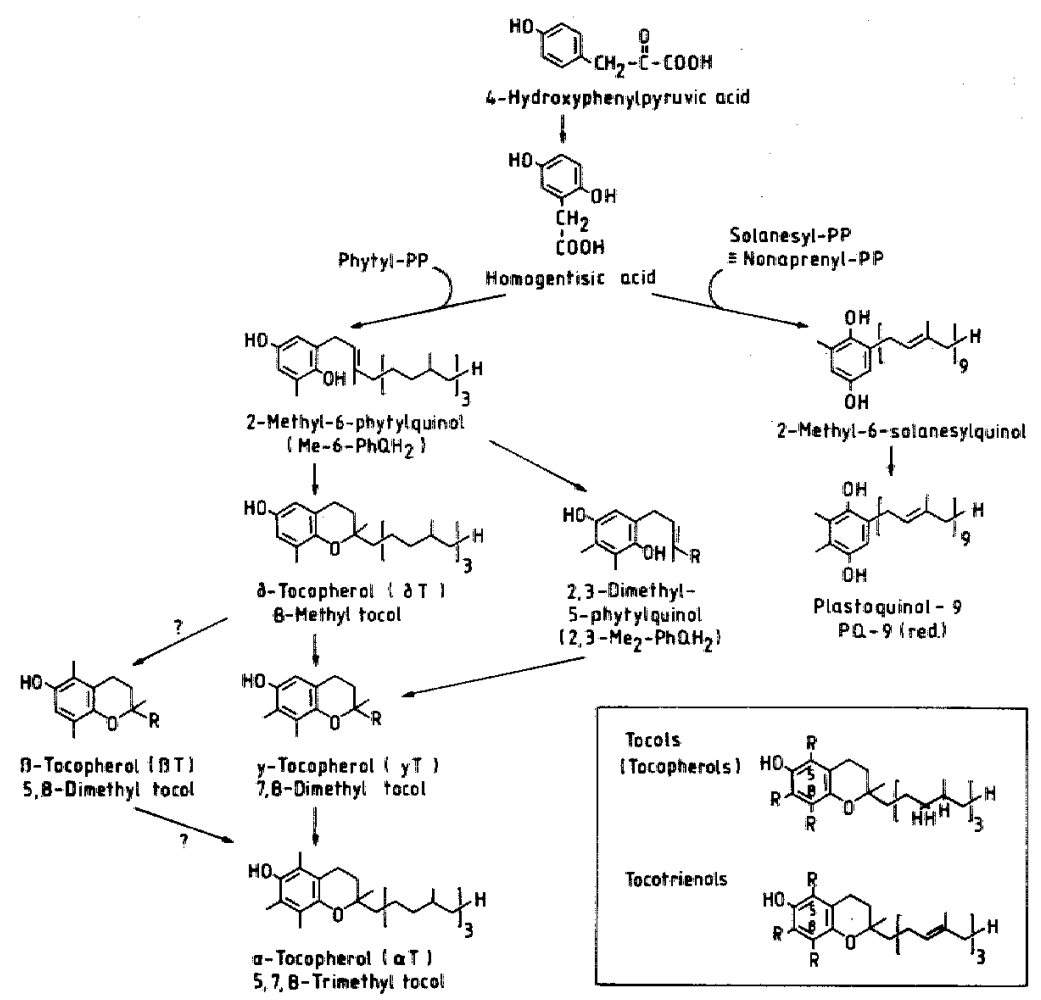

mase in chloroplasts of Nepeta cataria and spinach. Since the mevalonate-PP decarboxylase may be sensitive to the preparation procedure (see Arebalo and Mitchell 1984), in several cases IPP could not be recovered in feeding experiments with mevalonate (Kreuz and Kleinig 1981). On the other hand, synthesis of $\beta$-carotene in Phycomyces blakesleanus (for review see Goodwin 1983) and of sesquiterpenoid paniculide B in Andrographis paniculata (Anastasis et al. 1983) from leucine must be judged as side reactions. From the isotopic distribution it can be concluded that HMG-CoA formed from leucine undergoes cleavage, probably into acetoacetate and acetyl-CoA. These products are partially rearranged again to $\mathrm{HMG}-\mathrm{CoA}$.

\section{The hydrogenation steps in the synthesis of the phytyl moiety}

The stepwise hydrogenation of geranylgeranyl chlorophyllide $a$ to chlorophyll $a$ (= phytyl chlorophyllide $a$ ) via dihydro- and tetrahydrogeranylgeranyl chlorophyllide $a$ in chloroplasts was comprehensively studied by Rüdiger et al. (1977; Fig. 1c). Recently, Soll et al. (1983) demonstrated that two pathways for the hydrogenation of the geranylgeranyl moiety exist in chloro- plasts. In the chloroplast envelope membrane, geranylgeranyl-PP is hydrogenated to phytyl-PP, whereas in the thylakoid membrane the above reaction occurs with geranylgeranyl chlorophyllide $a$. NADPH functions as electron donor in both reactions.

The substrate specificity of enzymes involved in the $\alpha$ tocopherol and plastoquinone-9 synthesis

Both plastoquinone-9 as electron and proton carrier in the phosynthetic electron transport chain and $\alpha$-tocopherol as scavenger for energized oxygen species are formed from homogentisate and the corresponding prenyl-PP solely in chloroplasts (Schultz et al. 1981a; Fig. 1d and Fig. 2). From energetic considerations of organic chemistry, a multi-pathway-synthesis does not seem unrealistic (see Pennock 1983). However, studies on spinach chloroplasts have revealed that only one or two marked pathways exists in the synthesis of $\alpha$-tocopherol. All steps of its synthesis are localized at the inner chloroplast envelope membrane (Soll et al. 1984). The method to fractionate chloroplast membranes as developed by $\mathbf{R}$. Douce and coworkers (see Block et al. 1983) has been an important tool to study this.

The homogentisate-phytyl-PP prenyltransferase as 
the first enzyme of this pathway introduces solely phytyl-PP onto homogentisate (Fig. 2); geranylgeranyl$P P$ is inactive in this system. From the position isomers only 2-methyl-6-phytylquinol is formed; neither the 5nor the 3-phytyl isomer could be found (Soll et al. 1980b). Consequently, the pathway is directed in respect to substrate and position of the electrophilic substitution reaction at the aromatic nucleus.

In the following methylation step to yield the dimethyl compound, 2-methyl-6-phytylquinol is strongly preferred to its isomers, the 5- and the 3-phytyl compound (ratios are 100:10:5; Soll 1981). The main product is 2,3-dimethyl-5-phytylquinol. It is of interest that of the 2-methyl-6-prenylquinols, the corresponding 6geranylgeranyl compound (not occurring in chloroplasts) is preferred to the 6-phytyl compound (Soll et al. $1980 \mathrm{a})$. In the final methylation step $\gamma$-tocopherol is preferred to $\beta$-tocopherol $(100: 35)$ to yield $\alpha$-tocopherol.

A problem not yet sufficiently solved is the stage at which the cyclization reaction occurs to form the chromanol. Since the above experiments with intact chloroplasts supplied with 2-methyl-6-phytylquinol and S-adenosyl- $\mathrm{L}-\left[\right.$ methyl $\left.-{ }^{14} \mathrm{C}\right]$ methionine resulted in a decreasing ratio of labelling from 2,3-dimethyl-5-phytylquinol to $\gamma$ tocopherol and $\alpha$-tocopherol, it was inferred that the ring closure occurs at the stage of 2,3-dimethyl-5-phytylquinol. Although the ring closure to form $\gamma$-tocopherol could be demomstrated at this stage in intact chloroplasts (Soll and Schultz 1980) and in membranes of Capsicum chromoplasts (Camara et all 1982), experiments with envelope membrane fractions from chloroplasts (Soll et al. 1980b) need further verification.

Recently I: F. Pennock (personal communication) raised the question of why the cyclization does not occur at the stage of the 2-methyl-6-phytylquinol, since $\gamma$-tocopherol was recovered when $\delta$-tocopherol and S-adenosyl-L-[methyl $\left.{ }^{14}\right]$ methionine were applied to chloroplasts (Soll and Schultz 1980). The methylation rate of $\delta$-tocopherol to yield $\gamma$-tocopherol is still about half of that for 2-methyl-6-phytylquinol to form 2,3-dimethyl5-phytylcuinol (Soll 1981; Fig. 2).

Indeed, a more detailed inspection of the reaction reveals that 2-methyl-6-phytylquinol may undergo ring closure to yiell the corresponding chromanol $\delta$-tocopherol (see experiments in Fig. 3). When $\left[{ }^{3} \mathrm{H}\right]$-homogentisate and phytyl-PP were applied to intact chloroplasts in a slight hypotonic medium ("swollen chloroplasts"), 2-methyl-6-phytylquinol and the quinone (from the oxidation by air), and $\delta$-tocopherol as the cyclization product sequentially formed from the quinol, could be detected in the radioscan (Fig. 3; for details on a further experiment with $\left[1-{ }^{14} \mathrm{C}\right.$-phytyl-PP, see legend to the figure). Additionally, $\left[{ }^{3} \mathrm{H}\right]$-homogentisate was incorporated into plastoquinone-9 (or its demethyl compound 2-methyl-6-solanesylquinol; Fig. 2) at considerable rates (see also the next section). Light is not required in the prenylation and cyclization reactions.

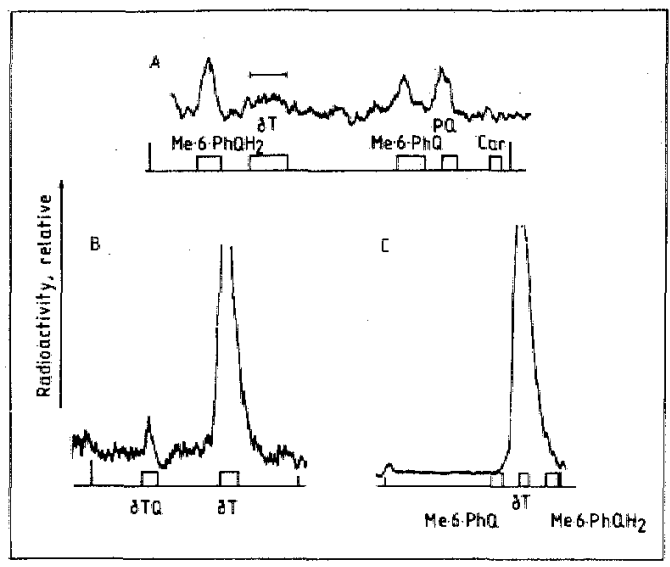

Fig. 3. Formation of 2-methyl-6-phytylquinol and quinone, $\delta$ tocopherol and plastoquinone-9 from $\left[{ }^{3} \mathrm{H}\right]$-homogertisate (1 $\mu M, 292 \mathrm{MBq}_{\mu \mathrm{mol}}^{-1}$; prepared as in Soll et al. 1980b) and phytyl-PP $(0.31 \mathrm{~m} M)$ in spinach chloroplasts. Chloroplasts were isolated as in Schulze-Siebert et al. (1984). They were suspended in a medium containing (in mM) sorbitol $100, \mathrm{~N}-2$ hydroxyethylpiperazine- $\mathrm{N}^{\prime}$-2-ethanesulfonic acid (adjusted with $\mathrm{KOH}$ to $\mathrm{pH} 7.6) 50, \mathrm{MgCl}_{2} 2, \mathrm{MnCl}_{2} 1, \mathrm{NaCl} 20, \mathrm{NaNO}_{3}$ $2, \mathrm{KH}_{2} \mathrm{PO}_{4} 0.5$, EDTA 1, ascorbate 5. A, Radioscan of TLC on silicagel with petrol ether (b.p. $60-80^{\circ}$ )/diethylether $3: 1$ as solvert. $B$, Radioscan of the re-chromatography of the $\delta$-tocopherol zone from (A) on silicagel with petrol ether/diethylether $1: 1$ as solvent. $C$, The same as in (B) on cellulose (impregnated with 7\% paraffin oil in petrol ether) with acetone/ wate $85: 15$ as solvent (reversed phase chromatography). Tocopherols and quimols were stained by $\mathrm{FeCl}_{3}$-bipyridine-reagent. To identify the position of the methylgroup, the nitroso derivative was formed according to Marcinkiewicz and Green (1959). The product formed coincided with the nitroso derivative of $\delta$-tocopherol (Janiszowska and Pennock 1976). Comparable results on the formation of $\delta$-tocopherol were obtained in another experiment by applying hormogentisate $(0.17 \mathrm{~m} M)$ and $\left[1{ }^{14} \mathrm{C}\right]$-phytyl-

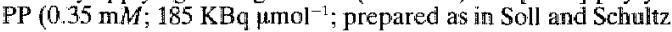
1981).

Summarizing the results on chloroplasts of spinach and Capsicum, the sequence in the formation of $\alpha$-tocopherol may be proposed as follows:

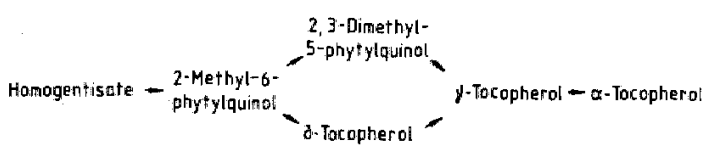

Furthermore $\beta$-tocopherol and $\beta$-tocotrienol (both 5,8-dimethylchromanol compounds occurring additionally to $\gamma$-tocopherol and $\gamma$-tocotrienol in cereals (for formula, see Fig. 2) are also suggested to originate from $\delta$ tocopherol and $\delta$-tocotrienol, respectively. There is only a demand on a prenyltransferase specific for geranylgeranyl-PP. As mentioned above, the methyltransferases in the subsequent reaction are not specific for the substrate and catalyze the reactions with phytyl- as well as with geranylgeranylquinols. 
The synthesis of plastoquinone-9 is also localized on the envelope membrane as shown by Soll et al. $(1980 \mathrm{~b}$, 1984). Homogentisate is prenylated with solanesyl-PP (= nonaprenyl-PP) to form specifically 2-methyl-6-solanesylquinol. The quinol is methylated by S-adenosylmethionine to form plastoquinol-9 (Figs $1 \mathrm{~b}$ and 2).

$\alpha$-Tocopherol as well as plastoquinone-9 and plastoquinol-9 are also constituents of the inner and outer chloroplast envelope membrane (total amount $\approx 10 \mathrm{mg}$ (g protein) $^{-1}$; Soll et all 1984).

\section{Plastidic reactions in phylloquinone (vitamin $K_{1}$ ) formation}

Of the vitamin $\mathrm{K}$ series especially the menaquinones are widely distributed among bacteria. In several gramnegative bacteria, menaquinones replace ubiquinones, particularly under anaerobic conditions, and transfer electrons from flavoprotein to reduce fumarate (for survey see Nicholls 1982). However, in chloroplasts a precise role of phylloquinone could not be found. Phylloquinone is present only in small amounts in photosystem I of chloroplast thylakoids [about $1 \mathrm{~mol}$ (100 mol chlorophyll $a)^{-1}$; Interschick-Niebler and Lichtenthaler 1981]. In contrast to menadione, 2-phytyl-1,4naphthoquinone and phylloquinone are completely inactive in cyclic photophosphorylation in spinach chloroplasts (F. Tiefenau, A. Bitsch and G. Schultz, unpublished results). The biosynthesis of menaquinones in bacteria has been studied in the laboratories of $R$. Bentley, I. G. Young and E. Leistner (for review see Bentley and Meganathan 1982). The pathway for the phylloquinone formation in plants seems to be similar if not identical (Figs 1e and 4).

Thomas and Threlfall (1974) have shown that shikimate is involved in the phylloquinone synthesis of maize seedlings. In bacteria, chorismate originating from the shikimate pathway condenses, presumably with succinylsemialdehyde energized by thiamine diphosphate, to yield 2-succinylbenzoate ( $=4$ - $\left(2^{\prime}\right.$-carboxyphenyl)-4-oxobutyrate) (Meganathan and Bentley 1981). The ring closure of the benzoate to form 1,4-dihydroxy-2-naphthoate (Meganathan et all 1980) occurs via the CoA-ester of 2-succinylbenzoate (Heide et al. 1982; E. Leistner, personal communication). Results on the cellular localization of 2-succinylbenzoate and 1,4-dihydroxy-2-haphthoate formation in higher plants are as yet lacking. In bacteria (Shineberg and Young 1976) as well as in spinach chloroplasts (solely in the envelope membranes; Schultz et al. 1981b) 1,4-dihydroxy-2naphthoate is prenylated by prenyl-PP to form the corresponding 2-prenyl-1,4-naphthoquinol. In the chloroplast reaction, phytyl-PP is preferred to geranylgeranylPP and farnesyl-PP. The optimal rate in the formation of the quinol is about $60 \mathrm{pmol}$ ( $\mathrm{mg}$ envelope protein) $)^{-1}$ $\mathrm{h}^{-1}$; the $\mathrm{K}_{\mathrm{m}}$ for the naphthoate is about $1 \mu M ; \mathrm{Mg}^{2+}$ is essential (optimal concentration $2 \mathrm{mM}$ ). 2-Phytyl-1,4naphthoquinone is a constituent of spinach chloroplasts

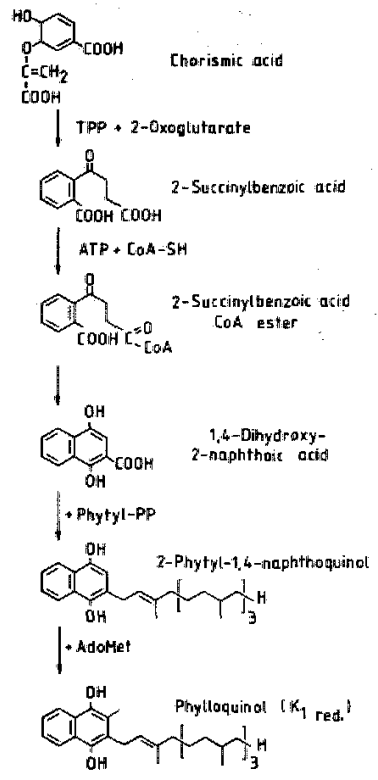

Fig. 4. Biosynthesis of phylloquinone (vitamin $\mathrm{K}_{\mathrm{y}}$ ) in thigher plants.

(McKenna et al. 1964). Degradative products of phylloquinone formed from radiolabelled 2-succinylbenzoate in maize shoots (Hutson and Threlfall 1980) indicate that the prenyl group is introduced to the carbon on which the carboxyl group was located; the latter is eliminated in a concerted reaction during prenylation. The final reaction in the phylloquinone formation is the

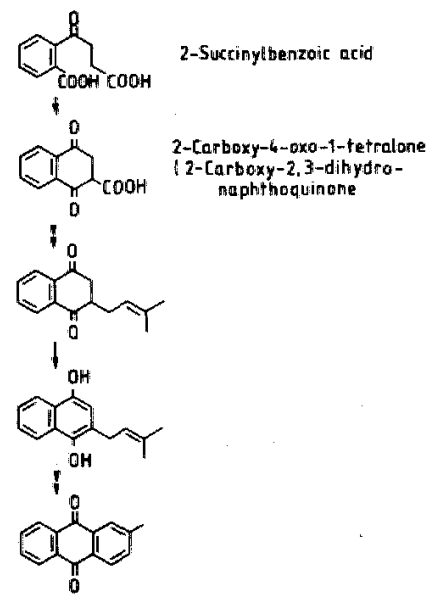

Fig. 5. Naphthoquinone and anthraquinone synthesis in Streptocarpus dunnii cell cultures via 2-carboxy-4-oxo-1-tetralone (Inoue et al. 1984b). 
methylation of the quinol by S-adenosylmethionine (Kaiping et al. 1984), which accurs at the chloroplast thylakoid membranes if a stroma fraction is simultaneously added (probably because of the supply of cofactors). The $\mathbb{K}_{\mathrm{m}}$ for S-adenosylmethionine is $3-5 \mu M$ : $\mathrm{S}$ adenosylhomocysteine acts as strong competitor; the $\mathbb{K}_{i}$ is $1-2 \mu M$.

The synthesis of the naphthalene nucleus in vitamin $K$ formation in bacteria and in chloroplasts exhibits only a restricted variability in the different steps. By contrast, the synthesis of the nucleus in the formation of naphthoquinones, anthraquinones, etc. in the secondary metabolism of some higher plants shows two modes of metabolism of 2-succinylbenzoate. 2-Succinylbenzoate may either form the "pre-aromatic" system of 2carboxy-4-oxo-1-tetralone (= 2-carboxy-2,3-dihydro1,4-naphthoquinone) (Inoue et al. 1984b; Fig. 5) or 1,4dihydroxy-2-naphthoate (Heide and Leistner 1981, Leistner 1981, Inoue et al. 1984a). Further metabolism of the tetralone and the naphthoate involves prenylation and decarboxylation reaction.

\section{Evolutionary significance}

Chloroplasts are organelles of high integrity not only in the energy conversion of light for photosynthetic $\mathrm{CO}_{2}-$ fixation, nitrate- and sulphate reduction, but also in metabolic pathways to synthesize lipids, essential amino acids, etc. Most, if not all, enzymes of these pathways are coded for in the nucleus (Kosmac and Feierabend 1984) and transferred across membranes into the chloroplast during processing. The question then arises why numerous of these pathways are placed in the plastidic organelle at the expense of genetic information of the nucleus. Obviously, it was of advantage during evolution that essential pathways became stabilized by maintaining a tight connection to the photosynthetic energy flow under all environmental conditions.

Acknowledgements - The author's experimental work mentioned in this paper was supported by the Deutsche Forschungsgemeinschaft.

\section{References}

Anastasis, P., Freer, I., Picken, D., Overton, K., Sadler, J. \& Singh. S. B. 1983. The role of leucine metabolism. Incorporation of $\left[2{ }^{13} \mathrm{C}\right]-$ and $\left[3-{ }^{13} \mathrm{C}\right]-$ leucine into sesquiterpenoid by tissue cultures of Andrographis paniculata. - J. Chem. Soc. Chem. Commun. 915: 1189-1191.

Arebalo, R. E. \& Mitchell, Jr., E. D. 1984. Cellular distribution of 3-hydroxy-3-methyl-glutaryl coenzyme A reductase and mevalonate kinase in leaves of Nepeta cataria. - Phytochemistry $23: 13-18$.

Bentley, R. \& Meganathan, R. 1982. Biosynthesis of vitamin $K$ (menaquinone) in bacteria. - Microbiol. Rev. 46: 241280.

Bickel, HI. \& Schultz, G. 1976. Biosynthesis of plastoquinone and $\beta$-carotene in isolated chloroplasts. - Phytochemistry 15: $1253-1255$.
- \& Schultz, G. 1979. Shikimate pathway regulation in suspension of intact chloroplasts. - Phytochemistry 18: 498499.

- ,Palme, L. \& Schultz, G. 1978. Incorporation of shikimate and other precursors into aromatic amino acids and prenylquinones of isolated spinach chloroplasts.. - Phytochemistry $17: 119-124$.

Block, M. A., Dorne, A. J., Joyard, J. \& Douce, R. 1983. Preparation and characterization of membrane fractions enriched in outer and inner membranes from spinach chloroplasts. 1. Electrophoretic and immunochemical analyses. - J. Biol. Chem. 258: 13273-13280.

Boudet, A. M., Graziana, A. \& Ranjewa, R. 1985. Recent advances in the regulation of the prearomatic pathway. - In The Biochemistry of Plant Phenolics (C. F. Van Sumere, ed.), Elsevier, Amsterdam. (In press).

Buchholz, B. \& Schultz, G. 1980 . Control of shikimate pathway in spinach chloroplasts by exogenous substrates. $-Z$. Plianzenphysiol. 100: 209-215.

Camara, B., Bardat, F., Seye, A., d'Harlingue, A. \& Monéger, R. 1982. Terpenoid metabolism in plastids. Localization of $a$-tocopherol synthesis in Capsicum chromoplasts. Plant Physiol. 70: 1562-1563.

Fiedler, E. Schultz, G. 1984. Purification of the associated 3dehydroquinate hydrolyase and shikimate oxidoreductase in spinach chloroplasts. - In Advances in Photosynthetic Research (C. Sybesma, ed.), Vol. III, pp. 893-896. Martinus Nijhoff/Dr. W. Junk, The Hague. ISBN 90-247-29440 .

- , Soll, J. \& Schultz, G. 1982. The formation of homogentisate in the biosynthesis of tocopherol and plastoquimone in spinach chloroplasts. - Planta 155: 511-515.

Goodwin, T. W. 1965. The biosynthesis of carotenoids. - In Biosynthetic Pathways in Higher Plants (J. B. Fridham and T. Swaim, eds), pp. 37-55. Academic Press, London. ISBN 65-26420.

- 1983. Developments in carotenoid biochemistry over 40 years. - Biochem. Soc. Trans. 11: 473-483.

Heidle L. \& Leistner, E. 1981. 2-Methoxy-carbonyl-3-prenyl1.4-maphthoquinone, a metabolite related to the biosynthesis of mollugin and anthraguinones in Galium mollugo $\mathrm{L}$. J. Chem. Soc. Chem. Commun. 1981: 334-336.

- Arendt, S. \& Leistner, E. 1982. Enzymatic synthesis, characterization and metabolism of the coenzyme A ester of o-succinylbenzoic acid, an intermediate in menaquinone (vitamin $\mathrm{K}_{2}$ ) biosynthesis. -J. Biol. Chem. 257: 7396-7400.

Heldt, H. W. \& Rapley, L. 1970. Specific transport of inorganic phosphate, 3-phosphoglycerate and dihydroxyacetone phosphate, and of dicarboxylates across the inner membrane of spinach chloroplasts. - FEBS Lett. 10: 143148.

Homeyer, U., Schulze-Siebert, D. \& Schultz, G. 1985. Control of pyruvate metabolism in spinach chloroplasts by exogenously added products and coenzymes. - J. Plant Physiol. (In press).

Hutson, K. G. \& Threlfall, D. R. 1980. Asymmetric incorporation of $4-(2$-carboxyphenyl)-4-oxobutyrate into phylloquinone by Zea mays. - Phytochemistry 19: 535-537.

Inoue, K., Shiobara, Y., Nayeohiro, H., Inouye, H., Wilson, G. \& Zenk, M. H. 1984a. Biosynthesis of anthraquinones and related compounds in Galium mollugo cell suspension cultures. - Phytochemistry 23" 307-311.

- Leda, S., Nayeshiro, H., Moritome, N. \& Inoruye, H. 1984b. Biosynthesis of naphthoquinones and anthraquinones in Streptocarpus dunnii cell cuiltures. - Phytochemistry 23: 313-318.

Interschick-Niebler, E. \& Lichtenthaler, H. K. 1981. Partition of phylloquinone $\mathrm{K}_{1}$ between digitonin particles and chlorophyll-proteins of chloroplast membranes from Nicotiana tabacum. - Z. Naturforsch. 36c: 276-283.

Janiszowska, W. \& Pennock, J.F. 1976. The biachemistry of vitamin E in plants. - Vitam. Horm. 34: 77-105. 
Kaiping, S., Soll, J. \& Schultz, G. 1984. Site of methylation of 2-phytyl-1,4-maphthoquimol in phylloquinone (vitamin $K_{1}$ ) synthesis in spinach chloroplasts. - Phytochemistry 23: 8991.

Kosmac, U. \& Feierabend, J. 1984. Synthesis of chloroplasts glycolipids and prenylquinones in $70 \mathrm{~S}$ ribosome-dieficient rye leaves and its relation to chlorophyll formation. - in Advances in Fhotosynthesis Research (C. Sybesma, ed.), Vol. IV, pp. 775-778. Martimus Nijhoff/Dr. W. Junk. The Hague. ISBN 90-247-2945-9

Kreuz, K. \& Kleinig, H. 1981. On the compartmentation of isopentenyl diphosphate synthesis and utilization in plant cells. - Planta 153: 578-581.

Leistner, E. 1981. Biosynthesis of plant quinones. $-I_{n}$ The Biochemistry of Plants (P. K. Stumpf and E. E. Conn, eds), Vol. 7, pp. 403-423. Acadenic Press, New York. ISBN 012-675407-1.

McKenna, M., Henninger, M. D. \& Crane, F. L. 1964. A secand naphthoquinone in spinach chloroplasts. - Nature 203 : $524-525$.

Marcinkiewicz, S. \& Green, J. 1959. The complete analysis of tocopherol mixtures. II. The separation of nitroso-tocopherols by paper chromatography and their determination. The Analysit 84: 304-312.

Meganathan, R. Bentley, R. 1981. Biosynthesis of o-succinylbenzoic acid in men- Escherichia coli mutant requires decarboxylation of $\mathrm{L}$-glutamate at the $\mathrm{C}-1$ position. - Biochemistry 20: $5336-5340$.

- Folger, T. \& Bentley, R 1980. Conversion of o-succinylbenzoate to dihydroxynaphthoate by extracts of Micrococcus luteus. - Biochemistry 19: 785-789.

Miflin, B. J. \& Lea, P. L. 1977. Amino acid metabolism. Annu. Rev. Plant Physiol 28: 299-329.

Murphy, D.J. \& Leech, R. M. 1978. The pathway of $\left[{ }^{14} \mathrm{C}\right]$ bicarbonate incorporation into lipids in isolated photosynthesising chloroplasts. - FEBS Lett. 88: 192-196.

Nicholls, $\mathbb{D} . \mathrm{G}$. 1982. Bioenergetics. An Introduction to Chemiosmotic theory. - Acadiemic Press, London. ISBN 0 12-518-120-5.

Pennock, J. F. 1983. The biosynthesis of chloroplastidic terpenoid guinones and chromanols. - Biochem. Soc. Trans. 11: $504-510$.

- Threlfall, D. R. 1983. Biosynthesis of ubiguinones and related compounds. - In Biosynthesis of Isoprenoid Compounds (J.W. Porter and S. L. Spurgeon, eds), Vol. 2, pp 191-303. John Wiley \& Sons, N.Y. ISBN 0-471-04807-0.

Roughan, G. \& Slack, R. 1984. Glycerolipid synthesis in leaves. - Trends Biochem. Sci. 9: 383-386.

Roughan, P. G., Holland, R., Slack, C. R. \& Mudd, J. B. 1978. Acetate is the preferred substrate for long-chain fatty acid synthesis in isolated spinach chloroplasts. - Biochem. J. $184: 565-569$.

Rüdiger, W., Hedden, P., Köst H. H. \& Chapman, D. J. 1977. Esterification of chlorophyllide by geranylgeranylpyrophosphate in a cell-free system from maize shoots. - Biochem. Biophys. Res. Commun. 74: 1268-1272.

Schulze-Siebert, D., Heineke, D., Scharf, H. \& Schultz, G. 1984. Pyruvate derived amino acids in spinach chloroplasts: Synthesis and its regulation during the photosynthesis. Plant Physiol. 76: 465-471.

Schulltz, G., Bickel, H., Buchholz, B. \& Soll, J. 1981a. The plastidic shikimate pathway and its role in the synthesis of plastoquinone-9, $a$-tocopherol and phylloquinone in spinach chloroplasts. - In Photosynthesis (G. Akoyumoglou, ed.), Vol. 5, pp: 311-318. Balaban International Science Services, Philadelphia. ISBN 0-86689-012-2.

- , Ellerbrock, B. H \& Soll, J. 1981b. Site of prenylation reaction in phylloquinone (vitamin $K_{1}$ ) synthesis of spinach chloroplasts. - Eur. J. Biochem. 117: 329-332.

-, Soll, J. \& Fiedler, E. 1982 . The biosynthesis of isoprenoid compounds in the chloroplast from the compartmental view. - In Biochemistry and Metabolism of Plant Lipids (J. F. G. M. Wintermans and P. J. C. Kuiper, eds), pp. 501505. Elsevier Biomedical Press, Amsterdam. ISBN 0-444$80457-9$.

Shineberg, B. \& Young. I. G. 1976. Biosynthesis of bacterial menaquinones: The membrane - associated 1,4-dihydroxy2-naphthoate octaprenyltransferase of Escherichia coli. Biochemistry 15: 2754-2758.

Soll, J. 1981 . Thesis, Univ. of Hannover, F,R.G

- \& Schultz, G. 1980. 2-methyl-6-phytylquinol and 2,3-dimethyl-5-phytylquinol as precursor of tocopherol synthesis in spinach chloroplasts. - Phytochemistry 19: 215-218.

- \& Schultz, G. 1981. Phytol synthesis from geranylgeraniol in spinach chloroplasts. - Biochem. Biophys. Res. Comman. 99: 907-912.

- , Douce, R. \& Schultz, G. 1980a. Site of biosynthesis of $\alpha-$ tocopherol in spinach chloroplasts. - FEBS Lett. 112: 243246.

- Kemmerling, M. \& Schultz, G. 1980b. Tocopherol and plastoquinone synthesis in spinach chloroplast subfractions. - Arch. Biochem. Biophys. 204: 544-550.

- , Schultz, G., Rüdiger, W. \& Benz, J. 1983. Hydrogenation of geranylgeraniol. Two pathways exist in spinach chloroplasts. - Plant Physiol. 71: 849-854.

- , Schultuz, G., Joyard, J., Douce, R. \& Block, M. A. 1984. Localisation and synthesis of prenylquinones in isolated outer and inner envelope membranes from spinach chloroplasts. - In Structure, Function and Metabolism of Plant Lipids (P. A. Siegenthaler and W. Eichenberger, eds), pp. 263-266. Elsevier, Amsterdam.

Spurgeon, S. L. \& Porter, J. W. 1983. Biosynthesis of carotemes. $-I n$ Biosynthesis of Isoprenoid Compounds, Vol. 2 (J. W. Porter and S. L. Spurgeon, eds), pp. 1-122. John Wiley, New York. ISBN 0-471-04807-0.

Stitt, M. \& ap Rees, T. 1979 . Capacities of pea chloroplasts to catalyse the oxidative pentose phosphate pathway and glycolysis. - Phytochemistry 18: 1905-1911.

Stumpf, P. K., Shikimata, T., Eastwell, K., Murphy, D. J., Liedvogel, B., Ohlrogge, J. B. \& Kuhn, D. N. 1982. Biosynthesis of fatty acids in a leaf cell. - In Biochemistry and Metabolism of Plant Lipids (J.F.G.M. Wintermans and P. J. C. Kuiper, eds), pp. 3-11. Elsevier, Amsterctam.

Thomas, G. \& Threlfall, D. R. 1974. Incorporation of shikimate and 4-(2'-carboxyphenyl)-4-oxobutyrate into phylloquinone. - Phytochemistry $13: 807-813$.

Threlfall, D. R. \& Whistance, G. R. 1971. Brosynthesis of isoprenoid quinones and chromanols. - In Aspects of Terpenoid chemistry and Biochemistry (T. W. Goodwin, ed.). pp. 357-404. Academic Press, London.

Whistance, G. R. \& Threlfall, D. R. 1970 . Homogentisic acid: precursor of plastoquinones, tocopherols and $\alpha$-tocopherolguinone in higher plants, green algae and blue green algae. - Biochem. J. 177: 593-600.

Williams, M. \& Randalt, D. D. 1979. Pyruvate dehydrogenase complex from chloroplasts of Pisum sativum L. - Plant Physiol. 64: 1099-1103. 